\title{
Clinical, anatomopathological, and immunohistochemical findings of a transitional cell carcinoma from nasal cavity, frontal and ethmoidal sinus with meningoencephalic invasion in a dog
}

\author{
Leonardo L. Gorza ${ }^{1 *}$, Ayisa R. Oliveira ${ }^{1}$, Maryleydy G. Porto ${ }^{2}$, Vania C. Santos ${ }^{2}$, Isabela Hardt ${ }^{3}$, \\ Luciana F. P. Maestri², Tayse D. Souza ${ }^{2}$ and Mayra C. Flecher ${ }^{2}$ \\ ${ }^{1}$ Department of Veterinary Medicine and Surgery, University of Minas Gerais, Belo Horizonte, Brazil \\ ${ }^{2}$ Department of Veterinary Medicine, University of Vila Velha, Vila Velha, Brazil \\ ${ }^{3}$ Autonomous Veterinary Professional, Vila Velha, Brazil
}

\begin{abstract}
Background: Primary neoplasms of the nasal cavity and sinuses are uncommon in domestic animals, most of which are of epithelial origin, being adenocarcinoma the most common tumor diagnosed in this region. Some malignant nasal cavity neoplasms may invade the brain causing clinical neurological signs, as well as purulent nasal secretion and epistaxis.

Case Description: A case of neoplasm is reported in a 14-year-old pincher presenting dyspnea, epistaxis, and neurological alterations. Necropsy revealed the presence of a mass in the oral cavity vestibule, and another in the whole nasal cavity with invasion of the cribiform plate, meninges and brain. Squamous cells carcinoma was diagnosed in the oral cavity and transitional carcinoma in the nasal cavity. The immunohistochemistry confirmed that the brain infiltration was of the same origin as the nasal cavity neoplasm.

Conclusion: The present report describes a rare case of transitional carcinoma of the nasal cavity as well as the frontal and ethmoidal sinuses with brain invasion, confirmed by immunohistochemistry. It is extremely important for veterinarians to include neoplasms in their differential diagnoses, when these animals show chronic respiratory signs and neurological alterations that do not improve with appropriate treatment, always associating with complementary exams, for correct diagnosis establishment and prognosis formulation.
\end{abstract}

Keywords: Canine, Epistaxis, Meninges, Nasal Tumor.

\section{Introduction}

Primary neoplasms of the nasal cavity and sinuses are uncommon in dogs (Wilson, 2017) and represents about $2 \%$ of all tumors in the species (Mukaratirwa et al., 2001; Wilson, 2017). The greatest risk for sinonasal tumor development is in animals with advanced age, between the ages of 10 and 15 years old (Wilson, 2017), and the etiology of these tumors is still not well established in veterinary medicine; however, some factors such as cigarette smoke and topical insecticides, are related to a higher risk of developing this condition (Reif et al., 1998). Most nasal neoplasms are of epithelial origin and malignant (Mukaratirwa et al., 2001), with adenocarcinoma being the most commonly diagnosed in this region, followed by transitional carcinoma, and less often, squamous cell carcinoma (Ninomiya et al., 2008).

Primary intracranial tumors are relatively common in dogs (Snyder et al., 2006; Wilson, 2017). Secondary intracranial neoplasms are classified into two categories, those that occur from metastases from distant locations and those that invade or compress the brain by extension of adjacent tissues. Among nasal neoplasms that lead to secondary intra-cranial invasion, carcinomas are the most commonly diagnosed tumor types (Snyder et al., 2008).

There are few recent reports on the occurrence of primary nasal neoplasms with subsequent brain invasion in dogs, as well as description of the cases' clinical findings (Lemetayer et al., 2017), preventing the establishment of more concise therapeutic protocols in these cases. Here, we describe an unusual case of transitional nasal carcinoma, of both frontal and ethmoidal sinuses, with meningoencephalic invasion in a dog, as well as squamous cell carcinoma of the oral cavity.

\section{Case Details}

A 14-year-old, neutered pincher was treated at the veterinary hospital Prof. Ricardo Alexandre Hippler, at University of Vila Velha (ES, Brazil). In the anamnesis, a history of lateral decubitus for 2 days, constant vocalization, walking in circles to the right, and motor incoordination was reported. The animal used phenobarbital to control seizures; however, the dose was not informed by the guardian. Physical 
examination revealed prostration, dyspnea, and nasal bleeding. During neurological examination, torcional nystagmus, ventrolateral strabismus in the right eye, and decreased facial sensitivity on the left side were detected. Complete blood count, biochemistry (Table 1), and a rapid canine distemper test (chromatographic immunoassay for the qualitative detection of $\mathrm{IgG}$ antibody) were requested. The hemogram showed normocytic normochromic anemia, leukocytosis $(66.900 / \mu \mathrm{l})$ due to neutrophilia (segmented cells $58.200 / \mu \mathrm{l})$, with a left shift (rods $669 / \mu \mathrm{l})$, in addition to lymphocytosis $(7.359 \mu \mathrm{l})$ and thrombocytosis $(582$ thousand $/ \mu 1)$. The biochemistry showed an increase in the level of alanine aminotransferase (ALT) (163.3 IU/1) and alkaline phosphatase (AP) (760.9 IU/1). The rapid distemper test was negative.

The treatment was stipulated with acetylcysteine syrup, benazepril associated with spironolactone, trimethoprim, and sulfamethoxazole, as well as maintenance of the phenobarbital; however, the animal died the next day. During necropsy, a whitish, solid, and infiltrative nodular lesion which projected from the upper vestibule of the oral cavity to the nasal cavity vestibule, was observed. After opening the nasal cavity, it was found that there was another mass extending to the dorsal nasal meatus, ethmoid cells and expanded to the ethmoid and frontal sinuses, entering the cribriform plate (Fig. 1). The opening of the skullcap revealed a mass in the middle of a large amount of necrotic material in the frontal region of the meninges and olfactory lobe.

The other organs did not present significant alterations. Multiple serial sections were performed from the beginning of the nasal cavity, covering the oral vestibule, nasal cavity, ethmoid sinuses, and brain. The removed fragments were processed through routine methods and stained with hematoxylin and eosin. In the histological transversal section of the nasal and oral cavity vestibules, a neoplastic proliferation of epithelial cells was observed, forming trabeculae and nests, infiltrating the lamina propria and oral cavity submucosa (Fig. 2a). The cells were large polyhedral cells with eosinophilic cytoplasms and evident desmosomal junctions, and high anisocariosis. In the centers of the nests, acantholytic squamous cells and corneal pearls characterizing an epidermoid carcinoma, were observed. In the innermost portion of the nasal cavity, covering the rostral region to the ethmoid sinus and in the cribriform plate there were epithelial neoplastic cells infiltrating the entire submucosa and extending to the adjacent bone tissue. The cells formed different sized nests surrounded by collagenous stroma. They were small, cubic to polyhedral shaped, with a small amount of cytoplasm, oval nucleus, with marked anisocariosis and evident nucleoli (Fig. 2b). In some areas, intense mitotic activity was observed and the lymphatic vessels throughout the nasal cavity were filled with neoplastic cell emboli. In the brain, the same neoplastic proliferation was observed in the meninges of the olfactory bulb, frontal, parietal, and occipital lobes as well as the cerebellum (Fig. 2c) and in the olfactory bulb and frontal lobe region the neoplasm infiltrated the neuropil. In order to confirm that the brain tumor was of an epithelial-transitional origin, immunohistochemistry of the meninges and the brain was performed. The result was positive for cytokeratin-7 (Fig. 2d).

Thus, focally extensive oral cavity squamous cell carcinoma and transitional carcinoma of the nasal cavity, frontal and ethimoidal sinuses with meningoencephalic invasion, was diagnosed.

Table 1. Complete blood count and biochemistry values of a 14-year-old pincher, afflicted by transitional cell carcinoma from nasal cavity, frontal and ethmoidal sinuses with brain invasion.

\begin{tabular}{lcc}
\hline \multicolumn{3}{c}{ Hemogram } \\
\hline Parameter & Reference values & Obtained values \\
\hline Hematocrit & $37 \%-55 \%$ & $32.2 \%$ \\
\hline Mean corpuscular volume (MCV) & $60-77 \mathrm{fl}$ & $73.2 \mathrm{fl}$ \\
\hline Mean corpuscular hemoglobin (MCH) & $30 \%-36 \%$ & $35.0 \%$ \\
& Leukogram & \\
Total leukocytes & $6,000-17,000 / \mu 1$ & $66.900 / \mu 1$ \\
Neutrophil & $3.00-11,500 / \mu 1$ & $58.200 / \mu 1$ \\
\hline Lymphocyte & $1.00-4,800 / \mu 1$ & $7.359 / \mu 1$ \\
Platelets & $175.000-500.00 / \mu 1$ & $582.000 / \mu 1$ \\
& Biochemistry & \\
Alanine aminotransferase (ALT) & $10-88 \mathrm{IU} / 1$ & $163.3 \mathrm{IU} / 1$ \\
Alkaline phosphatase (AP) & $44-147 \mathrm{IU} / 1$ & $760.9 \mathrm{IU} / 1$ \\
\hline
\end{tabular}



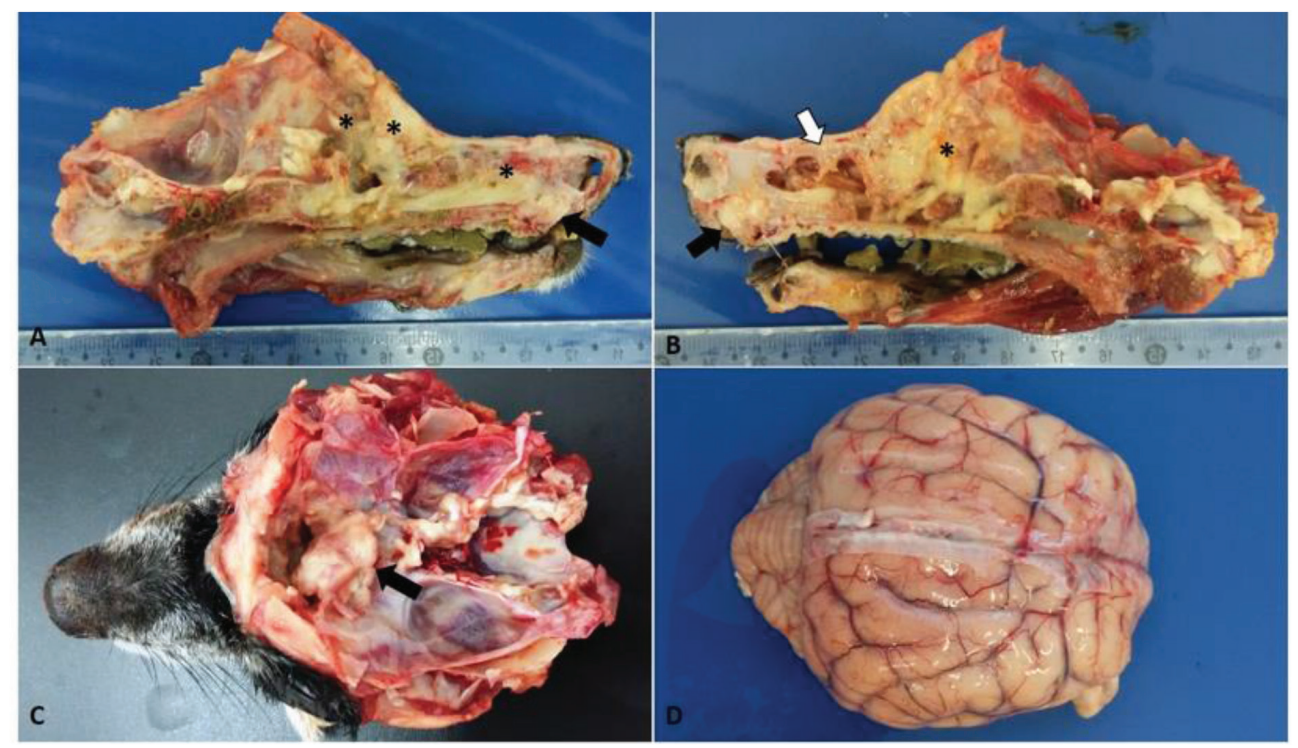

Fig. 1. Macroscopic findings in a 14-year-old dog with transitional nasal carcinoma with meningoencephalic invasion. (A) Sagittal cut of the face, right view: whitish mass originating from the oral vestibule invading the hard palate (black arrow) and yellowish white friable material observed at the beginning of the nasal cavity until the regions of ethmoid and frontal cells and sinuses $(*)$. (B) Sagittal cut of the face, left view: whitish mass originating from the oral vestibule invading the hard palate (black arrow); infiltrative mass associated with necrotic tissue that extended from the dorsal meatus of the nasal cavity (white arrow) to the cribriform plate ${ }^{*}$ ). (C) Cross sectioning of the skull. Whitish mass, cranial to the optical nerve in the olfactory bulb region showing the rupture of the cribriform plate due to the neoplasm. (D) Brain with moderate vessel disclosure.

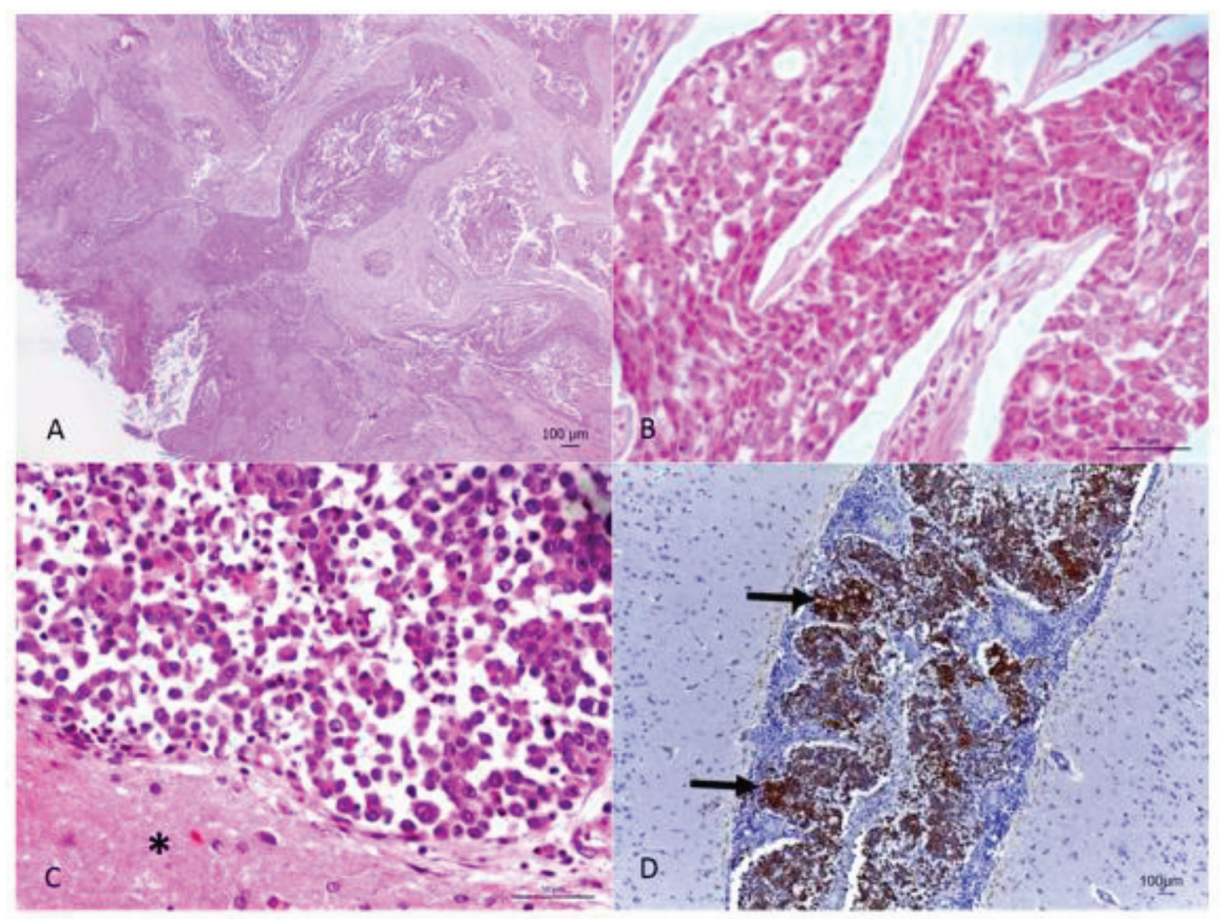

Fig. 2. (A) Oral neoplasm showing epithelial proliferation of the mucosa with papillary projection into the submucosa, forming trabeculae and nests with corneal pearls and acantholytic cells in the center. Hematoxylin and eosin. Scale bar= 100 micrometers. (B) Nasal cavity neoplasm, where rounded epithelial cell nests are observed showing eosinophilic cytoplasm, round nucleus and evident nucleolus. Hematoxylin and eosin. Scale bar $=50$ micrometers. (C) Meninges, adjacent to the brain tissue (*), with neoplastic infiltration from the transitional carcinoma. Hematoxylin and eosin. Scale bar $=50$ micrometers. (D) Marking for cytokeratin-7 in the neoplastic cells (arrow) in the meninges and vessels. Cytokeratin-7 immunolabeling. Scale bar $=100$ micrometers. 


\section{Ethical approval}

Data used in this manuscript were obtained from the Veterinary Hospital Prof. Ricardo Alexandre Hippler, at University of Vila Velha (ES, Brazil). This case was part of the clinical routine. Your necropsy and study were approved by the owner and hospital direction, respectively. All procedures were in accordance with institutional guidelines (University of Vila Velha).

\section{Discussion}

Although scarcely reported in the literature, it is concise that most primary nasal cavity and sinus neoplasms are malignant (Confer and de Paoli, 1978; Mukaratirwa et al., 2001; Ninomiya et al., 2008; Lemetayer et al.,, 2017). The reported dog had transitional cell carcinoma in the nasal cavity and sinus, this being the second most frequently diagnosed tumor in this location (Ninomiya et al., 2008). The clinical picture presented by animals affected with nasal neoplasms and brain invasion is extremely extensive. Epistaxis, mucopurulent nasal discharge, seizures, mydriasis, vestibular dysfunction, and blindness are some of the symptoms described (Confer and de Paoli, 1978; Rassnick et al., 2006; Snyder et al., 2008; Lemetayer et al., 2017). Facial deformity is a common finding since when affecting the nasal cavity and sinus, malignant epithelial neoplasms, in general, have an invasive behavior and lead to the destruction of the region's bone structures (Rassnick et al., 2006). In the present case, both signs related to the respiratory system as well as the nervous system were observed, ratifying the extensive symptoms presented, due to the fact that they cover two distinct sites. Necropsy and histopathological evaluation are of extreme importance in the diagnosis of the condition, as observed in the present case. However, immunohistochemistry, with antibodies for cytokeratin in general and cytokeratin 19 , is an important technique in aiding the diagnosis of neoplasms of epithelial origin (Ninomiya et al., 2008). The intense neutrophilia with a left shift evidenced in the reported dog, characterized a leukemoid reaction. This condition is frequently observed in dogs affected by malignant epithelial neoplasms (Sato et al., 2012), whereas this fact, associated with the nasal cavity's chronic inflammatory process, may justify the leukocytosis presented by the animal.

In conclusion, the present report describes a rare case of transitional carcinoma of the nasal cavity as well as the frontal and ethmoidal sinuses with brain invasion, confirmed by immunohistochemistry. Walking in circles, motor incoordination, right eye ventrolateral strabismus and decreased facial sensitivity on the left side were neurological signs presented by the animal. Dyspnea and epistaxis were evidenced respiratory signs. The presence of an epidermoid carcinoma in the same animal's oral cavity suggests how much domestic animals are exposed to carcinogens present in the urban environment. It is extremely important for veterinarians to include neoplasms in their differential diagnoses, when these animals show chronic respiratory signs and neurological alterations that do not improve with appropriate treatment, always associating with complementary exams, for correct diagnosis establishment and prognosis formulation.

\section{Conflict of interest}

The authors declare that they have no conflict of interest.

\section{Author's contribution}

Leonardo Gorza, Ayisa Oliveira, Isabela Hardt, and Mayra Flecher performed the necropsy and histopathological description. Luciana Maestri was the clinician of the case. Leonardo Gorza, Tayse Souza, Vania Santos, Maryleydy Porto, and Mayra Flecher wrote the manuscript. All authors reviewed the manuscript and made important contribution to the text.

\section{References}

Confer, A.W. and De Paoli, A. 1978. Primary neoplasms of the nasal cavity, paranasal sinuses and nasopharynx in the dog. A report of 16 cases from the files of the AFIP. Vet. Pathol. 15, 18-30.

Lemetayer, J., Al-Dissi, A., Tryon, K. and MacDonaldDickinson, V. 2017. Primary intranasal melanoma with brain invasion in a dog. Can. Vet. J. 58, 391396.

Mukaratirwa, S., Linde-Sipman, J.S. and Gruys, E. 2001. Feline nasal and paranasal sinus tumors: clinicopathological study, histomorphological description and diagnostic immunohistochemistry of 123 cases. J. Feline Med. Surg. 3, 235-245.

Ninomiya, F., Suzuki, S., Tanaka, H., Hayashi, S., Ozaki, K. and Narama, I. 2008. Nasal and paranasal adenocarcinomas with neuroendocrine differentiation in dogs. Vet. Pathol. 45, 181-187.

Rassnick, K.M., Goldkamp, C.E., Erb, H.N., Scrivani, P.V., Njaa, B.L., Gieger, T.L., Turek, M.M., McNiel, E.A., Proulx, D.R., Chun, R., Mauldin, G.E., Phillips, B.S. and Kristal, O. 2006. Evaluation of factors associated with survival in dogs with untreated nasal carcinomas: 139 cases (19932003). J. Am. Vet. Med. Assoc. 229, 401-406.

Reif, J.S., Bruns, C. and Lower, K.S. 1998. Cancer of the nasal cavity and paranasal sinuses and exposure to environmental tobacco smoke in pet dogs. Am. J. Epidemiol. 147, 488-492.

Sato, T., Shida, T., Maruo, T., Kawamura, H., Yamada, T., Ito, T., Takeda, H., Sugiyama, H., Ishikawa, T., Madarame, H., Kayanuma, H. and Suganuma, T. 2012. Primary lung carcinoma with paraneoplastic leukocytosis in a dog. J. Japan. Vet. Canc. Soc. 3, 20-24.

Snyder, J.M., Lipitz, L., Skorupski, K.A., Shofer, F.S. and van Winkle, T.J. 2008. Secondary intracranial neoplasia in the dog: 177 cases (1986-2003). J. Vet. Intern. Med. 22, 172-177. 
Snyder, J.M., Shofer, F.S., Van Winkle, T.J. and Massicotte, C. 2006. Canine intracranial primary neoplasia: 173 cases (1986-2003). J. Vet. Intern. Med. 20, 669-675.
Wilson, D.D. 2017. Tumors of the respiratory tract. In: Tumors in domestic animals. Ed., Meuten, D.J. Ames, Iowa: John Wiley \& Sons, pp: 365-370. 